\title{
HYPERPERFUSION SYNDROME - A REVIEW ARTICLE
}

\author{
*N. S. NEKI
}

\begin{abstract}
Hyperperfusion syndrome (HPS) is a clinical and neuro radiological entity characterized by headache, vomiting, altered mental status, blurred vision and seizures as well as images suggesting whitegray matter edema involving in most cases posterior regions of the brain, as demonstrated by magnetic resonance image. The development of HPS is most commonly associated with hypertensive encephalopathy, preeclampsia-eclampsia and hemolysis, elevated liver enzymes, low platelets (HELLP) syndrome, and immunosuppressive/cytotoxic drug. While elevated or relatively elevated blood pressure is common in many of these disorders, some hyperperfusion states such as calcineurininhibitor toxicity occur with no apparent pressure rise. In these cases, vasogenic edema is likely due primarily to dysfunction of the capillary endothelium itself, leading to breakdown of the blood-brain barrier. Modern imaging techniques and experimental models suggest that vasogenic edema is usually the primary process leading to neurologic dysfunction; therefore, prompt recognition and management of this condition should allow for clinical recovery if superimposed hemorrhage or infarction has not occurred.
\end{abstract}

Key words: Hyperperfusion Syndrome; Eclampsia; Altered sensorium.

\section{Introduction}

Hyperperfusion syndrome (HPS) is a neurotoxic state coupled with a unique CT or MR imaging appearance. It is a clinical-neuroradiological entity ${ }^{1}$ initially described in 1996 by Hinchey and co-workers, as reversible posterior leukoencephalopathy syndrome ${ }^{2}$. However, this term has fallen out of favor because none of its elements are completely accurate. The radiographic and clinical changes are not always reversible; the territory involved is not uniquely posterior; and gray matter may be affected as well, rather than purely white matter as the word "leukoencephalopathy" states. This syndrome is characterized by headache, visual disturbances, seizures, altered mental status and radiological findings of edema in the white matter of the brain areas perfused by the posterior brain circulation ${ }^{3}$.Various terminologies have been used to describe this condition, including "reversible posterior leukoencephalopathy syndrome" and "reversible posterior cerebral edema syndrome" among others ${ }^{4}$. Hypertension (HTN) is the most commonly identified cause of HPS, followed by medications, eclampsia and systemic factors. The pathophysiology of HTN related HPS is due to a failure of cerebrovascular autoregulation, which in turn results in vasogenic edema. Non-hypertensive HPS may be due to an autoimmune or immune response to various stimuli ${ }^{5}$. The pathology usually affects the posterior brain hemisphere (parietal-occipital region), which may be a consequence of reduced sympathetic innervation in this area.

The brain's autoregulatory capability successfully maintains a fairly stable cerebral blood flow in adults despite alterations in systemic mean arterial pressure (MAP) ranging from $50-150 \mathrm{~mm} \mathrm{Hg}$. In patients with chronic hypertension, this cerebral autoregulation curve is shifted, resulting in autoregulation working over a much higher range of pressures (e.g., $70-175 \mathrm{~mm} \mathrm{Hg}$ ). In these hypertensive patients, cerebral blood flow is kept steady at higher MAP, but a rapid lowering of pressure can more easily lead to ischemia on the lower end of the autoregulatory curve. This autoregulatory phenomenon is achieved through both myogenic and neurogenic influences causing small arterioles to contract and dilate. When the systemic blood pressure exceeds the limits of this mechanism, breakthrough of autoregulation occurs, resulting in hyperperfusion via increased cerebral blood flow, capillary leakage into the interstitium, and resulting edema. The predilection of all of the hyperperfusion disorders to affect the posterior rather than anterior portions of the brain may be due to a lower threshold for autoregulatory breakthrough in the posterior circulation.

Correspondence: Prof. N.S. Neki, Professor of Medicine, Govt. Medical College, Amritsar, India 


\section{Etiopathogenesis}

Table-I shows etiologies of HPS.

Table - I

Some Common Etiologies of Hyperperfusion Syndrome

Disorders in which increased capillary pressure dominates the pathophysiology

Hypertensive encephalopathy, including secondary causes such as renovascular hypertension, pheochromocytoma, cocaine use, etc.

Postcarotidendarterectomy syndrome

Preeclampsia/eclampsia

High-altitude cerebral edema

Disorders in which endothelial dysfunction dominates the pathophysiology

Calcineurin-inhibitor toxicity

Chemotherapeutic agent toxicity (e.g., cytarabine, azathioprine, 5-fluorouracil, cisplatin, methotrexate)

Glucocorticoids

Erythropoietin

HELLP syndrome (hemolysis, elevated liver enzyme levels, low platelet count)

Thrombotic thrombocytopenic purpura (TTP)

Hemolytic uremic syndrome (HUS)

Systemic lupus erythematosus (SLE)

Granulomatosis with polyangiitis (Wegener's)

While elevated or relatively elevated blood pressure is common in many of these disorders, some hyperperfusion states such as calcineurin-inhibitor toxicity occur with no apparent pressure rise. In these cases, vasogenice dema is likely due primarily to dysfunction of the capillary endothelium itself, leading to breakdown of the blood-brain barrier. It is useful to separate disorders of hyperperfusion into those caused primarily by increased pressure and those due mostly to endothelial dysfunction from a toxic or autoimmune etiology (Table-1). In reality, both of these pathophysiologic processes are likely playing some role in each of these disorders.

Some extremely common entities causing HPS include the following:

\section{Pregnancy-induced HPS:}

In HPS associated with pregnancy-induced HTN, pregnancy itself predisposes the brain to edema formation, particularly in late-pregnancy ${ }^{6}$. In some studies of pregnancy, the smooth muscle reactivity that leads to forced vasodilatation needs lower pressures to be produced ${ }^{7}$. For unknown reasons, in animals, in late-pregnancy state, there is a vasoconstriction activity in cerebral arteries in response to serotonin exposure, while the contrary occurs in non-pregnant specimens, in which the response results in vasodilatation ${ }^{8}$. Pregnancy causes direct effects on perivascular innervations particularly in pial vessels, producing hypertrophy of nerves. This suggests that neurotransmitters may have a role in hypertensive intolerance that causes HPS. It has been proposed that angiogenic factors may have a role in the prevention of HPS development, via diminishing endothelial dysfunction. Among endogenous antiangiogenic factors both soluble vascular endothelial growth factor (VEGF) receptor and placental growth factor (PIGF) are found increased in pregnancy 9,10 .

\section{Hypertension}

The compensatory mechanisms in the central nervous system (CNS) limit blood flow when there is a sudden increase in systemic BP, avoiding fluid leakage from the intravascular space to the interstitium. This autoregulatory mechanism is due to vasoconstriction as a result of an increase in sympathetic tone ${ }^{11}$. In HPS, this autoregulatory response is abnormal resulting in a breakdown of the normal blood brain barrier (BBB) and causing vasogenic brain edema ${ }^{12,13}$. There is an apparent predisposition for edema to occur in the posterior CNS areas, particularly in the occipitoparietal areas. This is because of the partial 
lack of sympathetic innervation of the vasculature that emerges from the basilar artery; however, other sites of the brain and cerebellum may be affected even when occipito-parietal areas are not involved $^{1,14,15,16}$, In non-hypertensive patients, cerebral blood flow is preserved while the mean arterial pressure (MAP) is maintained within a range of 60-120 mmHg. However, in patients with chronic HTN the auto-regulatory range is shifted to the left. Thus, while normotensive patients can develop HPS with an acute increase in BP rising to a MAP of 120 $\mathrm{mmHg}$, however among patients with chronic HTN the BBB disruption occurs with MAP levels greater than $150 \mathrm{mmHg}^{16}$.

\section{Drugs:}

The use of many drugs has been related with HPS. Many theories have been suggested for this strong drug-disease relation, including drug-induced HTN, nephrotoxicity, direct neurotoxicity, and endothelial damage ${ }^{17,18}$.

Many immunosuppressant drugs have been associated with the development of $\operatorname{HPS}^{19,20}$. Oxaliplatin has been reported as a possible cause of HPS. The possible explanation is its passage through the BBB with secondary fluid transudation and subsequent cerebral edema. Bevacizumab, a recombinant humanized monoclonal antibody, causes HPS by both increasing BP and inhibiting vascular endothelial growth factor (VEGF) ${ }^{21}$. Sunitinib is a tyrosine kinase inhibitor which also inhibits VEGF effects, via anti-VEGF receptor. Moreover, glucocorticoids such as dexamethasone have been shown to induce HPS, although it is an uncommon steroids-use complication, and thought to be related to HTN secondary to its mineralocorticoid effects ${ }^{22}$.

Vasopressive agents play a significant role in the development of acute new onset HTN, ultimately leading to HPS. Midodrine, a selective á-1 adrenoreceptor agonist can increase both venous and arterial constriction, thus may produce HTN-induced HPS ${ }^{19}$.

\section{Clinical features}

HPS commonly presents with seizures (74\%), altered sensorium or encephalopathy, headache and visual changes $^{23}$.Consciousness impairment may range in severity from confusion, somnolence, and lethargy to encephalopathy or coma. Other neurological features such as aphasia and sensory changes also occur. HPS can sometimes present similarly to CVA.

Visual disturbances may include blurred vision, cortical blindness and permanent visual field defects $^{24}$. Seizures, or status epilepticus (SE), may present as the initial clinical picture in some cases. Sluggish pupillary reflexes or frank miosis can be observed $^{25}$.

Brain stem involvement manifestations comprise dyspnea, anarthria, and dysphagia ${ }^{26}$. Memory disturbances and derangement of concentration may also occur. Intracranial HTN may be part of HPS ${ }^{27}$.

\section{Diagnosis}

1. Radiology 28

Computed tomography (CT) scan findings are negative in almost all cases of HPS and when positive, it is difficult to distinguish between HPS and acute stroke. Therefore the image study of choice is the magnetic resonance imaging $(\mathrm{MRI})^{29}$.
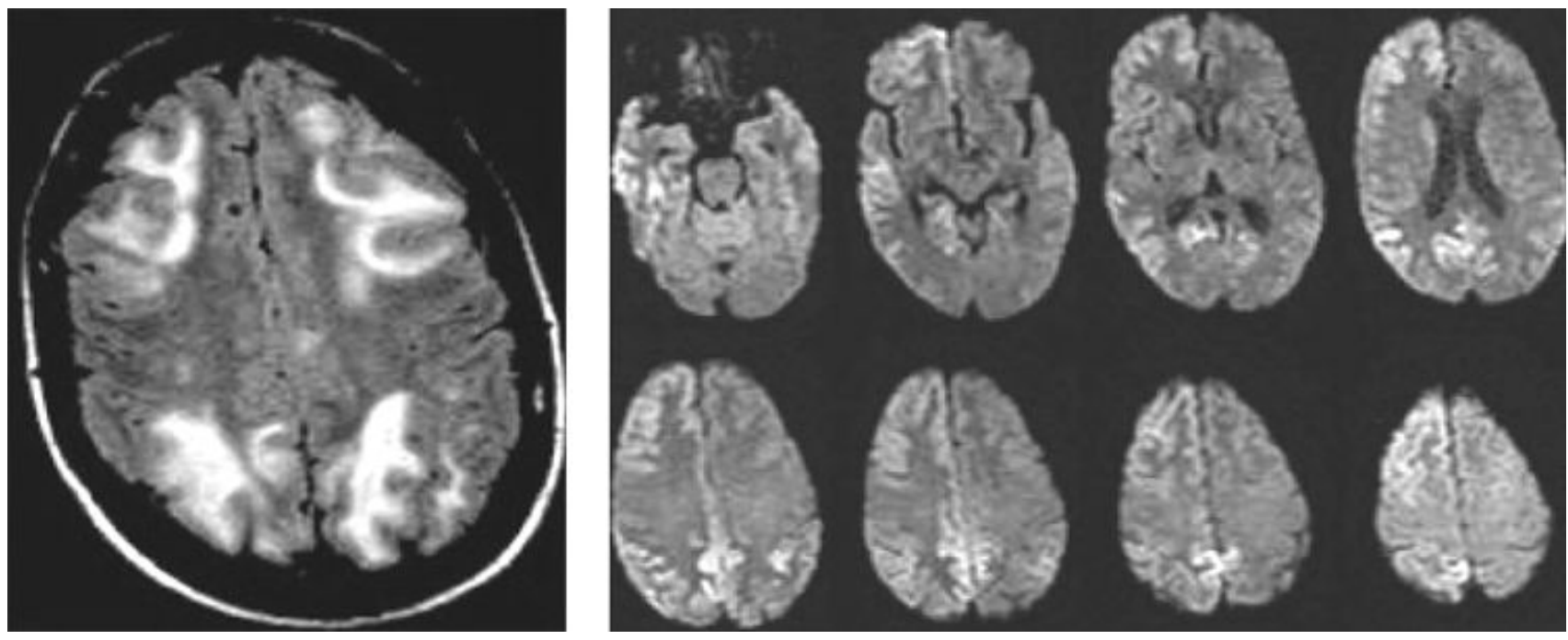

Fig. 1 \& 2 : bilateral and symmetric regions of edema typically located in the white matter 
There are four radiological patterns of HPS. Until recently, HPS was believed to consistently produce bilateral and symmetric regions of edema typically located in the white matter and predominating in the posterior parietal and occipital lobes. Holo hemispheric watershed pattern (23\%): Confluent vasogenic edema extends through the frontal, parietal, and occipital lobes. Involvement of the temporal lobes is less marked. Superior frontal sulcus pattern $(27$ $\%$ ): Patchy edema predominates in the frontal lobes along the superior frontal sulci.Dominant parietaloccipital pattern $(22 \%)$ : In this pattern previously thought to be typical of HPS, the posterior part of the parietal and occipital lobes is predominantly involved.Partial or asymmetric expression of the primary patterns $(28 \%)$.

\section{Cerebrospinal fluid analysis}

Invasive diagnostic methods such as lumbar puncture (LP) to evaluate cerebrospinal fluid (CSF) are not needed; however, in the setting of SLE or acquired immunodeficiency syndrome in which other causes of the neurological picture have to be ruled out, the CSF analysis serves as an important differential diagnostic tool ${ }^{30,31}$.

\section{Treatment}

General measures

Patients with HPS require the symptomatic measures usually taken in the ICU. Although most patients have stable hemodynamics, catecholamines are required occasionally. Upper airway protection is necessary in patients with impaired consciousness or ongoing seizure activity. Hypoglycemia should be looked for routinely and corrected. If glucose is given, $100 \mathrm{mg}$ of thiamine should be administered concomitantly. Patients should be routinely evaluated for hyperthermia and metabolic disturbance. These should be rapidly corrected. Aspiration pneumonia should be avoided.

\section{Control of blood pressure}

In hypertensive encephalopathy, MAP (Mean arterial pressure) should be reduced by $20-25 \%$ within the first $1-2 \mathrm{~h}$ or diastolic blood pressure reduced to 100 mmHg. More rapid lowering of blood pressure should be avoided, since it may worsen end organ function and cerebral infarction ${ }^{32,33}$. Intravenous therapy is generally preferred and drug choices include sodium nitroprusside, labetalol(which has both alpha and beta blocking activity) and calcium channel blockers. Angiotensin converting enzyme (ACE) inhibitors should be used cautiously in hypovolaemia and patients with underlying renal arterystenosis ${ }^{34}$.

\section{Control of seizures}

Seizures should be treated with anticonvulsant medication, and usually disappear once radiologic abnormalities have resolved ${ }^{35}$.Long-term antiepileptic treatment is not required. Immunosuppressant and cytotoxic drugs should be withdrawn or the dose reduced;and in those with Thrombotic thrombocytopenic purpura, plasmapheresis might be indicated ${ }^{36}$.Patients with persistent seizure activity should be given intravenous benzodiazepines ${ }^{37}$ (clonazepam $1 \mathrm{mg}$ or diazepam $10 \mathrm{mg}$ ). Patients with continuing seizure activity despite intravenous benzodiazepines should receive standard complementary intravenous anticonvulsant drugs (phenobarbital 10 to $15 \mathrm{mg} / \mathrm{kg}$, phenytoin $18 \mathrm{mg} / \mathrm{kg}$, or equivalent dose of fosphenytoin). Patients with refractory status epilepticus need midazolam, propofol or thiopental in titrated doses until remission of the clinical seizure activity ${ }^{38}$.

\section{Management during pregnancy}

In pre-eclampsia, delivery of the baby and placenta is the treatment of choice. Magnesium sulphate, which is superior to phenytoin and diazepam, is preferred for the treatment of seizures ${ }^{39}$. Antihypertensive drugs which might be used in pregnancy include methyldopa, hydralazine and labetalo1 ${ }^{40}$, whereas $\mathrm{ACE}$ Inhibitors are contraindicated, and atenolol might have adverse effects on placental function and fetal growth ${ }^{41}$.

\section{Conclusion}

Reversible posterior leukoencephalopathy syndrome typically presents as headache, visual loss and seizures, often in the setting of accelerated hypertension. Early diagnosis and treatment is essential. MRI is the investigation of choice and shows increased FLAIR and T2 signal predominantly in the posterior parietal and occipital lobes, with normal or decreased DWI signal, suggestive of vasogenic cerebral oedema. These findings are characteristic of this condition. This should be aggressively treated with anti-hypertensives, anticonvulsants and withdrawal of any immunosuppressant medication. This approach prevents progression to irreversible brain damage and cerebral infarction.

\section{References}

1. Lim MH, Kim DW, Cho HSet al. Isolated cerebellar reversible leukoencephalopathy syndrome in a patient with end stage renal disease. Intern Med 2008;47:43-50.

2. Hinchey J, Chaves C, Appignani Bet al. A reversible posterior leukoencephalopathy syndrome. N Engl J Med 1996;334:494-500. 
3. Kozak OS, Wijdicks EF, Manno EM et al. Status epilepticus as initial manifestation of posterior reversible encephalopathy syndrome. Neurology 2007;69:894-97.

4. FeskeSK.Posterior reversible encephalopathy syndrome: a review. SeminNeurol 2011, 31(2): 202-15.

5. Bartynski WS: Posterior reversible encephalopathy syndrome, part 2:controversies surrounding pathophysiology of vasogenicedema.AJNR Am J Neuroradiol 2008, 29(6):1043-1049.

6. Cipolla MJ. Cerebrovascular function in pregnancy and eclampsia. Hypertension 2007;50:14-24

7. Cipolla MJ, Vitullo L, McKinnon J. Cerebral artery reactivity changes during pregnancy and the postpartum period: a role in eclampsia? Am J Physiol Heart CircPhysiol; 2004, 286:H2 127-32.

8. Aukes AM, Vitullo L, Zeeman GG et al. Pregnancy prevents hypertensive remodeling and decreases myogenic reactivity in posterior cerebral arteries from Dahl salt sensitive rats: a role in eclampsia? Am J Physiol Heart CircPhysiol2007;292:H1071-6

9. Levine RJ, Maynard SE, QianCet al. Circulating angiogenic factors and the risk of preeclampsia. $\mathrm{N}$ Engl J Med 2004;350:672-83.

10. El Maalouf G, Mitry E, Lacout A et al. Isolated brainstem involvement in posterior reversible leukoencephalopathy induced by bevacizumab. J Neurol 2008; 255: 295-6

11. Yano Y, Kario K, Fukunaga $\mathrm{T}$ et al. A case of reversible posterior leukoencephalopathy syndrome caused by transient hypercoagulable state induced by infection. Hypertens Res 2005;28:619-23

12. Cipolla MJ. Cerebrovascular function in pregnancy and eclampsia. Hypertension 2007;50:14-24.

13. Kontos HA, Wei EP, NavariRMet al. Responses of cerebral arteries and arterioles to acute hypotension and hypertension. Am JPhysiol 1978;234:H371-83.

14. Doi Y, Kimura F, Fujiyama Tet al. Hypertensive brainstem encephalopathy without parieto-occipital lesion-two case reports. Neurol Med Chir (Tokyo) 2006;46:75-9.

15. Peng WX, Nakaii M, Matsushima T, Asakura H. Atypical case of reversible posterior

leucoencephalopathy syndrome associated with puerperal HELLP syndrome. Arch

GynecolObstet 2008;278:269-71.

16. Morelli N, Gori S, Michelassi MCet al. Atypical Hyperperfusionsyndromeinpuerperium. EurNeurol 2008;59:195-7.

17. Rajasekhar A, George TJ Jr. Gemcitabine-induced reversible posterior leukoencephalopathy syndrome: a case report and review of the literature. Oncologist 2007; 12:1332-5.
18. Yokobori S, Yokota H, Yamamoto Y. Pediatric posterior reversible leukoencephalopathy syndrome and NSAID-induced acute tubular interstitial nephritis. PediatrNeurol 2006;34:245-7

19. Kim JS, Lee KS, Lim SCet al. Reversible posterior leukoencephalopathy syndrome in a patient with multiple system atrophy: a possible association with oral midodrine treatment. MovDisord 2007;22:1043-6.

20. Pinedo DM, Shah-Khan F, Shah PC. Reversible posterior leukoencephalopathy syndrome associated with oxaliplatin. J ClinOncol 2007;25:5320-1.

21. El Maalouf G, Mitry E, Lacout A, Lièvre A, Rougier P. Isolated brainstem involvement in posterior reversible leukoencephalopathy induced by bevacizumab. J Neurol 2008;255:295-6

22. Irvin W, MacDonald G, Smith JK, Kim WY. Dexamethasone-induced posterior reversible encephalopathy syndrome. J ClinOncol 2007;25:2484-6.

23. Fugate JE, Claassen DO, Cloft HJ, Kallmes DF, Kozak OS, Rabinstein AA: Posterior reversible encephalopathy syndrome: associated clinical and radiologic findings.MayoClinProc 2010, 85(5):427-32.

24. Kwon S, Koo J, Lee S. Clinical spectrum of reversible posterior leukoencephalopathy syndrome. PediatrNeurol 2001;24:361-4.

25. E1 Karoui K, Le Quintrec M, DekeyserEet al. Hyperperfusionsyndromein systemic lupus erythematosus. Nephrol Dial Transplant 2008;23:757-63.

26. Prasad N, Gulati S, Gupta RKet al. Spectrum of radiological changes in hypertensive children with reversible posteriorleucoencephalopathy. $\mathrm{Br} \mathrm{J}$ Radiol 2007;80:422-9

27. Tanioka R, Yamamoto $Y$, Sakai $M$ et al. Convalescence of atypical reversible posterior leukoencephalopathy syndrome in human immunodeficiency virus infection. J Med Invest 2007;54:191-4.

28. Bartynski WS, Boardman JF (2007) Distinct imaging patterns and lesion distribution inposterior reversible encephalopathy syndrome. AJNR Am J Neuroradiol 28: 1320-27.

29. Sweany JM, Bartynski WS, Boardman JF. "Recurrent" posterior reversible encephalopathy syndrome: report of 3 casesHPS can strike twice! J Comput Assist Tomogr 2007;31:148-56.

30. Mak A, Chan BP, YehIBet al. Neuropsychiatric lupus and reversible posterior leucoencephalopathy syndrome: a challenging clinical dilemma. Rheumatology (Oxford) 2008;47:256-62.

31. Saeed MU, Dacuycuy MA, Kennedy DJ. Hyperperfusionsyndromein HIV patients: case report and review of the literature. AIDS 2007;21:781-82. 
32. Ramsay LE, Williams B, Johnston GD, et al. British Hypertension Society guidelinesfor hypertension management 1999: summary. BMJ 319: 630-35.

33. Varon J, Marik PE. Clinical review: the management of hypertensive crises. CritCare2003; 7: $374-84$.

34. Vaughan CJ, Delanty N. Hypertensive emergencies. Lancet2000; 356: 411-17.

35. Garg RK. Posterior leukoencephalopathy syndrome. Postgrad Med J2001; 77: 24-28.

36. Bakshi R, Bates VE, Mechtler LL, Kinkek PR, Kinkel WR. Occipital lobe seizures as the major clinical manifestation of reversible posterior leukoencephalopathy syndrome: magnetic resonance imaging findings. Epilepsia1998; 39: 295-99.
37. Bakshi R, Shaikh ZA, Bates VE, Kinkel PR. Thrombotic thrombocytopenic purpura. Brain CT and MRI findings in 12 patients. Neurology 1999; 52: $1285-88$.

38. Meierkord H, Boon P, Engelsen B, et al. EFNS guideline on the management of status epilepticus in adults. Eur J Neurol 17: 348 -55.

39. Sibai BM. Treatment of hypertension in pregnant women. N Engl J Med1996; 335: 257-65.

40. Varon J, Marik PE. Clinical review: the management of hypertensive crises. CritCare2003; 7: $374-84$.

41. Buch J. Urapidil, a dual-acting antihypertensive agent: Current usage considerations. AdvTher 2010; 27: $426-43$. 